\title{
Emotion, Cognition, and Behavior
}

\section{R. J. Dolan}

Emotion is central to the quality and range of everyday human experience. The neurobiological substrates of human emotion are now attracting increasing interest within the neurosciences motivated, to a considerable extent, by advances in functional neuroimaging techniques. An emerging theme is the question of how emotion interacts with and influences other domains of cognition, in particular attention, memory, and reasoning. The psychological consequences and mechanisms underlying the emotional modulation of cognition provide the focus of this article.

A $\mathrm{n}$ ability to ascribe value to events in the world, a product of evolutionary selective processes, is evident across phylogeny (1). Value in this sense refers to an organism's facility to sense whether events in its environment are more or less desirable. Within this framework, emotions represent complex psychological and physiological states that, to a greater or lesser degree, index occurrences of value. It follows that the range of emotions to which an organism is susceptible will, to a high degree, reflect on the complexity of its adaptive niche. In higher order primates, in particular humans, this involves adaptive demands of physical, sociocultural, and interpersonal contexts.

The importance of emotion to the variety of human experience is evident in that what we notice and remember is not the mundane but events that evoke feelings of joy, sorrow, pleasure, and pain. Emotion provides the principal currency in human relationships as well as the motivational force for what is best and worst in human behavior. Emotion exerts a powerful influence on reason and, in ways neither understood nor systematically researched, contributes to the fixation of belief. A lack of emotional equilibrium underpins most human unhappiness and is a common denominator across the entire range of mental disorders from neuroses to psychoses, as seen, for example, in obsessive-compulsive disorder (OCD) and schizophrenia. More than any other species, we are beneficiaries and victims of a wealth of emotional experience.

In this article I discuss recent developments in the study of human emotion where, for example, a neurobiological account of fear, anger, or disgust is an increasingly urgent goal. Progress in emotion research mirrors wider advances in cognitive neurosciences where the idea of the brain as an information processing system provides a highly influential metaphor. An observation

Wellcome Department of Imaging Neuroscience, Institute of Neurology, Queen Square, London WC1N 3BG, UK. E-mail: r.dolan@fil.ion.ucl.ac.uk by the 19th-century psychologist, William James, questions the ultimate utility of a purely mind-based approach to human emotion. James surmised that "if we fancy some strong emotion, and then try to abstract from our consciousness of it all the feelings of its bodily symptoms, we find we have nothing left behind, no mind-stuff out of which the emotion can be constituted, and that a cold and neutral state of intellectual perception is all that remains" (2). This quotation highlights the fact that emotions as psychological experiences have unique qualities, and it is worth considering what these are. First, unlike most psychological states emotions are embodied and manifest in uniquely recognizable, and stereotyped, behavioral patterns of facial expression, comportment, and autonomic arousal. Second, they are less susceptible to our intentions than other psychological states insofar as they are often triggered, in the words of James, "in advance of, and often in direct opposition of our deliberate reason concerning them" (2). Finally, and most importantly, emotions are less encapsulated than other psychological states as evident in their global effects on virtually all aspects of cognition. This is exemplified in the fact that when we are sad the world seems less bright, we struggle to concentrate, and we become selective in what we recall. These latter aspects of emotion and their influences on other psychological functions are addressed here.

\section{Emotion, Perception, and Attention}

An evolutionary perspective on emotion suggests that environmental events of value should be susceptible to preferential perceptual processing. One means of achieving this is by emotion enhancing attention, leading to increased detection of emotional events. The influence of emotion on attention can be studied in classic visual search and spatial orienting tasks. In a visual search, the standard finding is that the time taken to detect a specified target increases in direct proportion to the number of irrelevant distracters, indi- cating serial attentive processing. However, for emotional stimuli there is more rapid target detection for faces with positive or negative expressions, or for spiders or snakes, with the most consistent capture of attention being evident for fear-relevant stimuli (3). Similar effects are seen in spatial orienting tasks where there is a faster response to targets appearing on the same side as an emotional cue (e.g., faces, spiders, threat words, conditioned shapes) and a slower response to those appearing on the opposite side $(4,5)$. Neuroimaging data, using spatial orienting paradigms, point to orbital prefrontal cortex as a possible site of interaction (4).

The "capture of attention" is not the sole means by which emotional stimuli influence perception, and emerging evidence indicates mechanisms independent of attention. Perceptual processing under conditions of limited attention as, for example, processing of stimuli at unattended spatial locations is often referred to as preattentive. In visual backward masking paradigms, a briefly presented (ms) target can be rendered invisible if immediately followed by a second "masking stimulus." In situations where the hidden target stimulus is an emotional item, for example a conditioned angry face or a spider, preserved processing can be indexed by differential skin conductance responses (SCRs) to fear-relevant compared with fear-irrelevant targets, even though the target stimulus is not perceived (6). Similar findings are evident using the attentional blink paradigm. This refers to a situation where detection of an initial target stimulus in a visual stimulus stream leads to impaired awareness, or "inattentional blindness," for a successive second target. This inattentional blindness is greatly diminished where a second target is an emotional item (7). This finding suggests an advantage in detection of an emotional item even in circumstances where attentional resources are limited.

Studies of patients with focal brain lesions provide additional evidence for independence of emotional processing from attentional mechanisms. After brain damage to right inferior parietal cortex, patients frequently fail to perceive a stimulus presented in their contralesional hemifield (spatial neglect) or, in milder forms, fail to perceive a stimulus when a simultaneous stimulus is presented on the ipsilesional side (sensory extinction). This contralesional deficit is greatly attenuated for emotional stimuli, such as faces with happy or angry expressions (8) or images of spiders (9). Noncon 


\section{SCIENCE'S COMPASS}

scious processing of emotion has also been demonstrated in the blindfield of patients with damage to primary visual cortex (10). These findings indicate processing of emotional stimuli occurs before the operation of selective attention and such "preattentive processing" results in enhanced stimulus detection.

Pre-attentive processing of emotional stimuli, such as faces, implies an early discrimination between the occurrence of emotional and nonemotional events. Using magneto-encephalography (MEG), dis-

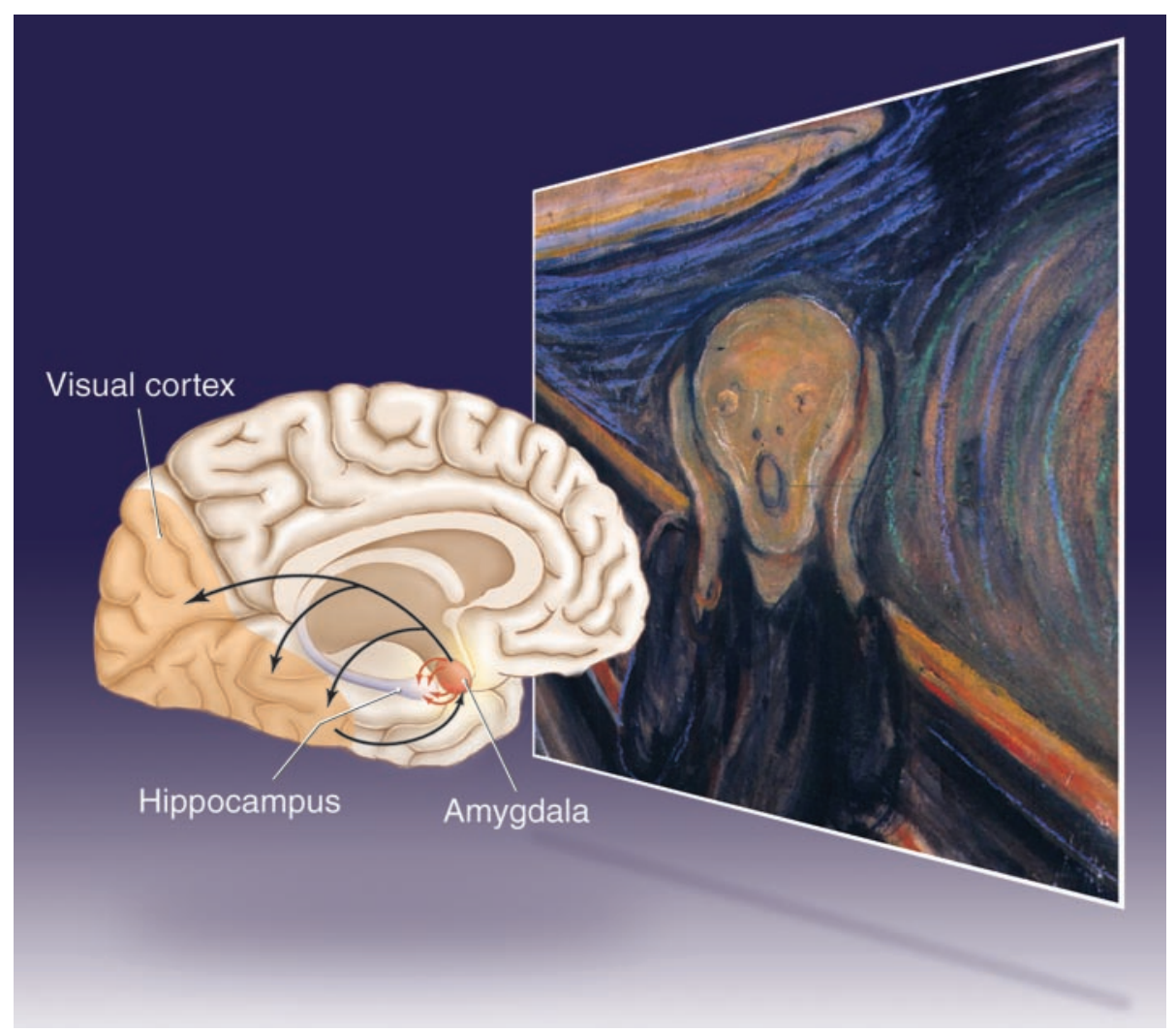

Fig. 1. An emotional-perceptual-memory circuit in the human brain. The amygdala (red), an anterior medial temporal lobe structure, is a crucial structure in registering emotional occurrences. Extensive connection (arrows) to visual cortex (orange) and hippocampus (blue) allows amygdala to modulate their function and facilitate perceptual and memory functions in those regions.

criminatory responses to emotional faces are seen in midline occipital cortex as early as 100 to $120 \mathrm{~ms}$ after stimulus onset, before the onset of a characteristic face-related response at approximately $170 \mathrm{~ms}(11,12)$. Intermodal binding of emotion for presentation of anger in voice and face is associated with a distinct electroencephalographic potential occurring at about $100 \mathrm{~ms}$ (13). Short-latency responses (120 to $160 \mathrm{~ms}$ ) to aversive stimulus presentation are also seen during direct intracerebral recordings within ventral prefrontal cortex (14). Thus, electrophysiological data point to rapid and widespread neuronal responses to emotional stimuli that precede responses associated with actual stimulus identification which occur at approximately $170 \mathrm{~ms}$ after stimulus onset.

An important neurobiological question is how processing of emotional stimuli proceeds in the absence of attention. Accumulating evidence points to the amygdala as an important mediator of emotional influences on perception (Fig. 1). In functional neuroimaging experiments using visual backward masking paradigms, where emotional stimuli are presented out of awareness, an amygdala response discriminates between unseen emotional and unseen nonemotional targets $(15,16)$. In other experiments with overt stimulus presentation but example coarse visual cues present in fearful faces, can be processed by a noncortical pathway to enable rapid adaptive responses to danger (20).

A related neurobiological question is how preattentive processing of emotional events influences, and indeed enhances, perception. One possibility is that inputs from emotional processing regions, in particular the amygdala, modulate the function of regions involved in early object perceptual processing. Anatomically, the amygdala receives visual inputs from ventral visual pathways and sends feedback projections to all processing stages within this pathway (21). Neuroimaging data provide evidence for context-dependent enhancement of functional connectivity between amygdala and extrastriate visual regions expressed during processing of an emotional visual input $(22,23)$. There is now evidence showing this connectivity has psychological consequences in that after amygdala damage a visual perceptual enhancement for emotional items is abolished (7).

\section{Emotion, Memory, and Learning}

Privileged perceptual processing of emotional events provide a means of not only indexing occurrences of value but facilitating their availability to other cognitive domains. The cognitive domain where the influence of emotion is best understood is memory. Enhanced memory for events of value allow better predictions regarding biologically important occurrences when re-encountering similar events in the future. The best example is seen in classical conditioning, which provides an inflexible, ubiquitously expressed form of emotional memory. In simple terms, this form of memory describes a situation where a neutral stimulus, through pairing in temporal contiguity with an emotional stimulus (for example, an aversive noise in fear conditioning), acquires an ability to predict future occurrences of this emotional event. From a human behavioral perspective, the importance of this form of memory is that it provides a potential link between a psychological mechanism and psychopathological conditions, such as phobias and post-traumatic stress disorder (PTSD).

Studies demonstrate that human amygdala is critical for fear conditioning, a form of implicit memory. Patients with amygdala damage do not acquire conditioned fear responses despite retaining explicit knowledge regarding the conditioned (CS) and unconditioned stimulus (UCS) associations (24). In contrast, patients with hippocampal damage and intact amygdala preserve fear conditioning despite being unable to demonstrate explicit knowledge regarding CS-UCS contingencies (25). Functional neuroimaging experiments also confirm the importance of the 


\section{SCIENCE'S COMPASS}

amygdala for learning of CS-UCS associations but point to a time-limited role for this structure $(26,27)$. Evidence that the role of the amygdala during emotional learning may be time-limited could indicate that more enduring memory effects are expressed elsewhere in the brain. Although there is an emphasis on the role of the amygdala in human fear conditioning studies, evidence indicates that it supports other forms of associative learning, including reward and appetitive learning $(28,29)$.

Enhanced autobiographical or explicit memory for emotional events is well documented in anecdotal accounts of enhanced recollection for events such as the assassination of President Kennedy or the Challenger shuttle disaster. The benefit of emotion on episodic memory function is confirmed in numerous studies showing mnemonic enhancement for material that encompasses personal autobiographical, picture-, and word-based items $(30,31)$, an effect most pronounced in free recall tasks. An enhanced memory for emotional items is also reported in amnesiacs, who despite profound deficits in episodic memory show normal memory enhancement for emotional material when tested by recognition (32).

A striking feature of the biology of emotional memory is a dependence on the amygdala that transcends the implicit-explicit distinction. Thus, patients with bilateral amygdala damage do not show an advantage in subsequent recall of emotional items and events $(33,34)$. The critical role of the amygdala is also evidenced by functional neuroimaging experiments where engagement of the amygdala during encoding predicts later recall of emotional material (35, 36 ). Crucially, enhanced amygdala activity to both positive and negative stimuli is predictive of later memory (31). The role of the amygdala in episodic memory extends beyond encoding processes, as evidenced by the fact that this structure is also engaged during retrieval of emotional items and contexts $(37,38)$.

The neurochemical mechanisms by which emotional events augment memory have been extensively studied in animal experiments that provide evidence of a $\beta$-adrenergic modulation (39). Emotional memory enhancement in human subjects can be blocked by administration of the $\beta$-adrenoreceptor blocker propranolol before study (40). This blockade is equivalent to that seen after human amygdala damage, providing indirect evidence that the amygdala might be a critical locus for propranolol's effects.

The fact that amygdala is engaged during episodic recall for emotional material suggests a role beyond providing a neuromodulatory signal to extra-amygdala structures at encoding. Psychological evidence that emotion influences episodic memory function indicates influences on hippocampal function and most probably extra-amygdala regions. Learningrelated plastic changes have been extensively described in animal studies of emotion as, for example, the experience-dependent retuning of sensory cortices after conditioning (41). These plastic changes may also be important in the expression of emotional memory in humans. For example, auditory cortex plasticity during fear conditioning to tones can be demonstrated with neuroimaging while administration of the central muscaranic receptor blocker scopolamine, before conditioning, blocks its expression (42). This finding is consistent with animal studies, implicating amygdala influences on cholinergic neurotransmission in the establishment of enduring memory traces (41).

\section{Emotion and Subjective Feeling States}

One problem that confronts human emotion research is a conflation of mechanisms indexing the occurrence of an emotional event, which may include automatic response repertoires, referred to as emotion, and their subjective or experiential counterparts, referred to as feelings $(43,44)$. Feelings are defined as mental representations of physiological changes that characterize and are consequent upon processing emotion-eliciting objects or states. In more extended form, the suggestion is that patterned neural responses provide for a differentiation of feeling states, this account assigns an important causal role to afferent feedback, sensory and neurochemical, to the brain regarding emotioninduced changes in body state. The importance of afferent feedback in the experience of emotion is supported by phenomenological evidence from patients with a rare acquired failure of peripheral autonomic regulation, pure autonomic failure (PAF), who have subtle blunting of emotional experience. However, the role of feeling states extends beyond providing subjective coloring to experience, and it is proposed that feelings influence functions such as decision making and interpersonal interactions (43).

A consequence of the fractionation of emotion and feelings implies the following functional arrangement. Perception of emotional events leads to rapid, automatic, and stereotyped emotional responses that contrasts with more long-term modulatory behavioral influences mediated by feeling states. If this general scheme is correct, then it is expected that brain systems supporting emotional perception and execution should be distinct from those supporting feeling states. It has been proposed that structures mediating feeling states are those that receive inputs regarding the internal milieu, viscera and musculoskeletal
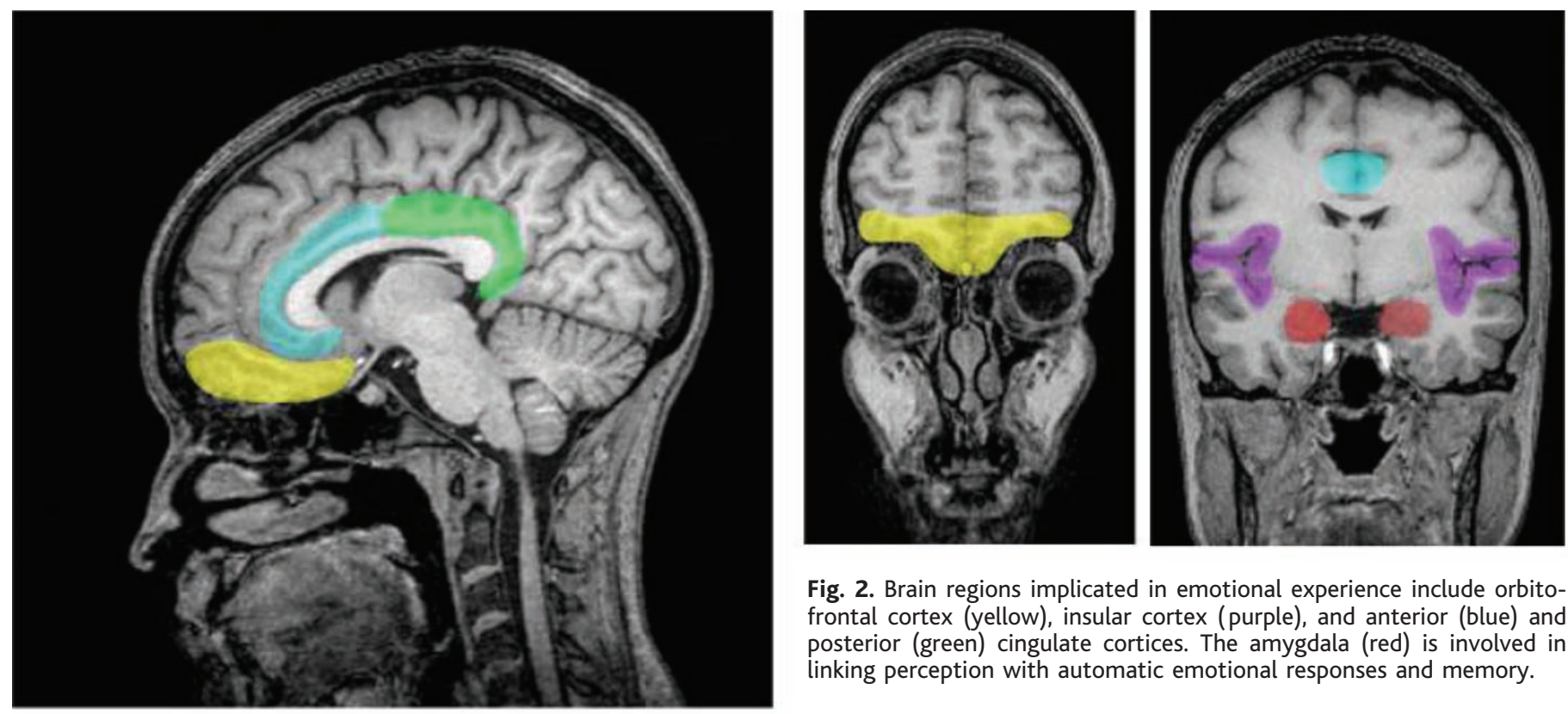

Fig. 2. Brain regions implicated in emotional experience include orbitofrontal cortex (yellow), insular cortex (purple), and anterior (blue) and posterior (green) cingulate cortices. The amygdala (red) is involved in linking perception with automatic emotional responses and memory. 


\section{SCIENCE'S COMPASS}

structures and include the brain stem tegmentum, hypothalamus, insula, and somatosensory and cingulate cortices (43).

There is now accumulating evidence that emotion and feeling are mediated by distinct neuronal systems. Functional neuroimaging experiments indicate that the generation and representation of peripheral autonomic states involve many of the predicted structures, particularly anterior cingulate and insularsomatosensory cortices (45-47) (Fig. 2). More specifically, recall of subjective feeling states associated with past emotional experiences engages regions encompassing upper brainstem nuclei, hypothalamus, somatosensory, insula and orbitofrontal cortices (48). In subjects with PAF, absence of visceral afferent information regarding the peripheral body state attenuates emotion and effort-related activity in similar regions $(49,50)$. A notable feature of these studies is an absence of amygdala engagement, a critical structure in emotional perception. Indeed a study of patients with unilateral and bilateral amygdala lesions indicates that they experience no deficit in their phenomenal experience of emotion (51). The implication is that there is a segregation within emotion-processing regions between those mediating perceptualmnemonic and experiential effects.

\section{Emotion and Decision-Making}

Within philosophy there is a long tradition that views emotion and reason in direct opposition. Such an oppositional relation has been questioned on the basis that, under certain circumstances, emotion-related processes can advantageously bias judgment and reason. This biasing effect appears to reflect influences of perceptual emotional mechanisms on the one hand and feeling states on the other. In terms of the former, neuropsychology and functional neuroimaging evidence indicate that the amygdala contributes to perceptual value judgments as, for example, making trustworthy decisions in relation to the facial appearance of others $(52,53)$. In terms of the latter, psychological data point to subtle influences of body states on decision making. For example, in masked presentation of either fear-conditioned or nonconditioned stimuli, subjects show differential shock expectancy ratings on shock versus noshock trials despite their lack of awareness of shock-predictive stimuli (54). Individuals who are able to detect their heartbeat on a heartbeat detection task, an index of visceral awareness, have enhanced performance predicting the likely occurrence of a shock in these same paradigms (55). The inference here is that better predictive judgments are mediated via an enhanced awareness of bodily states of arousal.

The volitional control of behavior is dependent on the functions of prefrontal cortex, particularly its dorsolateral and dorsomedial sectors. An emotional contribution to high-level decision making is evident after ventromedial prefrontal cortex damage, which may have no consequence for intellectual function but results in patients making personally disadvantageous decisions (56). The proposal is that these subjects fail to evoke appropriate feeling states associated with the contemplation of possible scenarios which constitute options for action. As formulated in the somatic marker hypothesis, this region provides access to feeling states in relation to past decisions during contemplation of future decisions of a similar nature (44). Thus, evocation of past feeling states biases the decision-making process, toward or away from a particular behavioral option. Empirical support for the theory includes evidence that patients with lesions to ventromedial prefrontal cortex fail to generate the normal anticipatory SCR responses in tasks where they ponder potentially risky choices (57). In addition, neuroimaging and neuropsycholgical evidence indicate that this region is activated during anticipatory states $(46,58)$ and by outcomes associated with reward or punishment (59). The functions of this region may also extend beyond this to include a role in regulating interpersonal interactions by providing the basis for what the philosopher Suzanne Langer described as the "involuntary breach of selfhood" that constitutes empathic experience. A lack of capacity for empathy may account for behavioral deficits of a sociopathic nature seen in subjects with acquired ventromedial prefrontal damage $(60,61)$.

\section{Conclusions}

A growing interest in the neurobiology of emotion parallels a wider recognition of its importance to human experience and behavior. The broad outlines of brain structures that mediate emotion and feelings are now reasonably clear and include brainstem autoregulatory systems; amygdala, insula, and other somatosensory cortices; cingulate and orbital-prefrontal cortices. Within this set of brain regions there is variable contribution to perceptual, mnemonic, behavioral, and experiential aspects of emotion. Despite progress in defining a functional anatomy of emotion, we still have little idea how emotion relates to other major axes of affective experience represented by motivation and mood. This is an issue that is critical to a deep understanding of many psychiatric disorders. For example, patients with mood disorders display dysfunction in similar brain regions to those that mediate emotion, yet at a psychological level the nature of the relation is far from clear $(62,63)$. Furthermore, how neurochemical control systems modulate affective states, including emotional states, is largely unknown. There is also the perplexing issue of how emotion infects rational thought processes such that people adhere, often with great conviction, to ideas and beliefs that have no basis in reason or reality. Lastly, there is an urgent need to examine the role of emotion in cognitive develop- ment and, in particular, to address how the growth of emotional awareness informs mechanisms that underwrite the emergence of selfidentity and social competence.

\section{References and Notes}

1. K. J. Friston et al., Neuroscience 59, 229 (1994).

2. W. James, The Principles of Psychology (Holt, New York, 1890).

3. A. Ohman et al., J. Exp. Psychol. Gen. 130, 466 (2001).

4. J. L. Armony et al., Neuropsychologia 40, 817 (2002)

5. K. Mogg et al., Behav. Res. Ther. 35, 297 (1997).

6. F. Esteves et al., Psychophysiology 31, 375 (1994).

7. A. K. Anderson, E. A. Phelps, Nature 411, 305 (2001).

8. P. Vuilleumier et al., Neurology 56, 153 (2001).

9. Neuroreport 12, 1119. (2001)

10. B. de Gelder et al., Neuroreport 10, 3759 (1999).

11. E. Halgren et al., Cereb. Cortex 10, 69 (2000).

12. M. Eimer, A. Holmes, Neuroreport 13, 427(2002).

13. G. Pourtois et al., Neuroreprort 11, 1329 (2000).

14. H. Kawasaki et al., Nature Neurosci. 4, 15 (2001).

15. J. S. Morris et al., Nature 393, 467 (1998).

16. P. J. Whalen et al., J. Neurosci. 18, 411 (1998).

17. P. Vuilleumier et al., Neuron 30, 829 (2001).

18. J. S. Morris et al., Brain 124, 1241 (2001).

19. P. Vuilleumier et al., Neuropsychologia 40, 2156 (2002).

20. J. LeDoux, The Emotional Brain (Weidenfeld \& Nicholson, London, 1998).

21. D. G. Amaral, J. L. Price, A. Pitkanen, S. T. Carmichael, in The Amygdala: Neurobiological Aspects of Emotion, Memory and Mental Dysfunction, J. Aggleton, Ed. (Wiley-Liss, New York, 1992).

22. J. S. Morris et al., Brain 121, 47(1998).

23. P. Rotshtein et al., Neuron 32, 747 (2001).

24. K. S. LaBar et al., J Neurosci 15, 6846 (1995).

25. A. Bechara et al., Science 269, 1115 (1995).

26. C. Buchel et al., Neuron 20, 947 (1998).

27. K. S. LaBar et al., Neuron 20, 937 (1998).

28. I. S. Johnsrude et al., J Neurosci 20, 2649(2000).

29. J. O'Doherty et al., Neuron 33, 815 (2002).

30. E. A. Phelps et al., Brain Cogn. 35, 85 (1997).

31. S. B. Hamann et al., Nature Neurosci. 2, 289 (1999).

32. S. B. Hamann et al., Learn. Mem. 4, 301 (1997).

33. R. Babinsky et al., Behav. Neurobiol. 6, 167 (1993).

34. R. Adolphs et al., Learn. Mem. 4, 291 (1997).

35. L. Cahill et al., Proc. Natl. Acad. Sci. U.S.A. 93, 8016 (1996).

36. T. Canli et al., J. Neurosci. 20, RC99 (2000).

37. E. J. Maratos et al., Neuropsychologia 39, 910 (2001).

38. R. J. Dolan et al., Neuroimage 11, 203 (2000).

39. J. L. McGaugh, Science 287, 248 (2000).

40. L. Cahill et al., Nature 371, 702 (1994).

41. N. M. Weinberger, Neurobiol. Learn. Mem. 70, 226 (1998).

42. C. Theil, K. J. Friston, R. J. Dolan, Neuron 35, 567 (2002).

43. A. Damasio, The Feeling of What Happens (Harcourt Brace, New York, 1999).

44. A. R. Damasio, Descartes' Error (Picador, London, 1995).

45. H. D. Critchley et al., Brain 124, 1003 (2001).

46. H. D. Critchley et al., Neuron 29, 537 (2001).

47. H. D. Critchley et al., J. Neurosci. 20, 3033 (2000).

48. A. R. Damasio et al., Nature Neurosci. 3, 1049 (2000).

49. H. D. Critchley et al., Nature Neurosci. 4, 207 (2001).

50. _ Neuron 33, 653 (2002).

51. A. K. Anderson et al., J. Cogn. Neurosci. 14, 70 (2002).

52. R. Adolphs et al., Nature 393, 470 (1998).

53. J. S. Winston et al., Nature Neurosci. 5, 277 (2002).

54. A. Ohman et al., J. Exp. Psychol. Gen. 127, 69 (1998).

55. E. S. Katkin et al., Psychol. Sci. 12, 366 (2001).

56. A. Bechara et al., Cereb. Cortex 10, 295 (2000).

57. A. Bechara et al., Brain 123, 2189 (2000).

58. A. Bechara et al., Science 275, 1293 (1997).

59. R. D. Rogers et al., J. Neurosci. 19, 9029 (1999).

60. S. W. Anderson et al., Nature Neurosci. 2, 1032 (1999).

61. R. J. Davidson et al., Science 289, 591 (2000).

62. H. S. Mayberg et al., Biol. Psych. 48, 830 (2000).

63. W. C. Drevets, Progr. Brain Res. 126, 413 (2000).

64. R. J. D. is supported by a Wellcome Trust Programme Grant. 